\title{
Electroantennogram responses of an invasive species fall webworm (Hyphantria cunea) to host volatile compounds
}

\author{
TANG Rui ${ }^{1,2}$, SU MaoWen ${ }^{1,2} \&$ ZHANG ZhongNing ${ }^{1 *}$ \\ ${ }^{1}$ State Key Laboratory of Integrated Management of Pest Insects and Rodents, Institute of Zoology, Chinese Academy of Sciences, Beijing 100101, \\ China; \\ ${ }^{2}$ Graduate University of Chinese Academy of Sciences, Beijing 100049, China
}

Received March 1, 2012; accepted June 11, 2012; published online August 1, 2012

\begin{abstract}
Electroantennogram responses of fall webworms (Hyphantria cunea) to 55 host volatile compounds were conducted. Results showed that male moths were more responsive to trans-2-hexenyl acetate, nonanal, hexanal, trans-2-hexenal, hexanol, isoamyl acetate, acetic acid cis-3-hexenyl ester, ethyl acetoacetate, citronellal and (+)-citronellal, and females were more responsive to hexanol, hexanal, acetophenone, isoamyl acetate, nonanal, trans-2-hexenal, acetic acid cis-3-hexenyl ester, citronellal, cis-3-hexen1-ol and trans-2-hexen-1-ol. Seven bioactive compounds were also selected for further dosage response experiments, and a positive correlation was found between the fall webworm and the tested compounds. A maximum dosage level of $1000 \mu \mathrm{g}$ was tested and found to elicit significantly higher activity from male moths compared with lower $(1,10,100 \mu \mathrm{g})$ concentrations.
\end{abstract}

Hyphantria cunea, electroantennogram analysis, botanical secondary substances, invasive pest

Citation: Tang R, Su M W, Zhang Z N. Electroantennogram responses of an invasive species fall webworm (Hyphantria cunea) to host volatile compounds. Chin Sci Bull, 2012, 57: 4560-4568, doi: 10.1007/s11434-012-5356-Z

Host plants play an important role in the mating and reproduction of defoliating insects, including through influencing the behavior, communication and mechanisms of pheromone signals [1]. For example, some insects use chemicals from host plants as precursors for the biosynthesis of sex pheromones [2]. Certain insects are also known to release sex pheromones only when in the presence of odors from their host plants [3]. Through influencing insect behavior, host volatiles play an important role in courtship and breeding within insect mating systems.

Botanical chemicals, such as plant volatiles, can often enhance the bioactivity of insect sex pheromones. For example, one of the most common green leaf volatiles, $(Z)-3$ hexene acetate, can enhance the effects of sex pheromones in Helicoverpa zea [4] and Heliothis virescens [5]. Similarly, blending green leaf volatiles with sex pheromones of Cydia pomonella resulted in more male moths being caught compared with controls of only sex pheromone compounds [6].

*Corresponding author (email: zhangzn@ioz.ac.cn)
Hyphantria cunea, a global quarantine pest belonging to the Arctiidae family of Lepidoptera, has caused extensive destruction in China since its invasion in 1979. Although a number of host plants are used by $H$. cunea, the insects mostly feed on mulberry (Morus alba L.), rock maple (Acer saccharum), poplar (Populus tremula) and ailanthus (Ailanthus altissima) [7]. Identification of $H$. cunea sex pheromone compounds began in the early 1970s, and today, 4 primary components of $H$. cunea are known around the world [8]. Chemical composition of the sex pheromones of $H$. cunea in China was first reported in 2008 [7]. Interactions between host plants and insects through semiochemicals are now an important subject for chemical ecologists [9]. However, there remains little published work examining relationships between $H$. cunea and plant odors [10]. Different botanical odors may differentially affect the ability of $H$. cunea to locate host plants, which may consequently influence their feeding. Based on published work of Lepidoptera bioactive compounds [11] and host leaf volatile components [12], we identified 55 chemicals that could 
potentially influence Lepidoptera insects and conducted electrophysiological experiments to elucidate the effect of allelochemicals on $H$. cunea and its invasive mechanisms in China.

\section{Materials and methods}

\subsection{Insects}

Larvae were collected in May 2009 in Sanhe of Hebei Province, China. Larvae were raised in the laboratory with mulberry leaves under conditions of $16 \mathrm{~L}: 8 \mathrm{D}$ and $25^{\circ} \mathrm{C}$. Pupae were sexed, separated by gender, and raised under the same conditions as larvae until eclosion. Newly emerged adult moths were housed at room temperature and 1-3 d moths were used in electrophysiological analysis.

\subsection{Chemicals for electroantennogram}

Chemicals were selected from published work according to their bioactive effect on moths. Their sources and levels of purity are shown in Table 1.

Table 1 Source and purity of chemicals used in EAG studies

\begin{tabular}{|c|c|c|c|c|c|}
\hline Chemicals & Purity $(\%)$ & Source $^{a)}$ & Chemicals & Purity(\%) & Source $^{a)}$ \\
\hline Alcohols & & & $(-)-\beta$-Pinene & $\geqslant 99$ & $\mathrm{a}$ \\
\hline 3-Butyn-1-ol & $\geqslant 97$ & $\mathrm{~b}$ & $(+)-\alpha$-Pinene & $\geqslant 99$ & a \\
\hline Butanol & $\geqslant 97$ & $\mathrm{~d}$ & $(-)$ - $\alpha$-Pinene & $\geqslant 99$ & a \\
\hline 1,4-Pentadien-3-ol & $\geqslant 97$ & $\mathrm{c}$ & $( \pm)$-3-Carene & $\geqslant 99$ & $\mathrm{a}$ \\
\hline cis-3-Hexen-1-ol & $\geqslant 99$ & $\mathrm{a}$ & $\beta$-Caryophyllene & $\geqslant 97$ & $\mathrm{c}$ \\
\hline trans-2-Hexen-1-ol & $\geqslant 99$ & $\mathrm{a}$ & Esters & & \\
\hline 2-Ethyl-1-hexanol & $\geqslant 99$ & a & Ethylacetoacetate & $\geqslant 97$ & $\mathrm{~d}$ \\
\hline 2,5-Hexanediol & $\geqslant 96$ & $\mathrm{c}$ & Isoamylacetate & $\geqslant 97$ & $\mathrm{~d}$ \\
\hline Hexanol & $\geqslant 97$ & $\mathrm{~d}$ & Aceticacidcis-3-hexenylester & $\geqslant 99$ & $\mathrm{e}$ \\
\hline Heptanol & $\geqslant 97$ & $\mathrm{~d}$ & trans-2-Hexenylacetate & $\geqslant 99$ & $\mathrm{e}$ \\
\hline Octanol & $\geqslant 97$ & d & Ketones & & \\
\hline Decanol & $\geqslant 97$ & $\mathrm{~d}$ & 3-Pentanone & $\geqslant 97$ & $\mathrm{~b}$ \\
\hline cis,cis-9,12-Octadecadienol & $\geqslant 95$ & $\mathrm{~b}$ & (+)-Camphor & $\geqslant 97$ & $\mathrm{~b}$ \\
\hline Nerolidol & $\geqslant 95$ & $\mathrm{~b}$ & (-)-Camphor & $\geqslant 97$ & $\mathrm{~b}$ \\
\hline Geraniol & $\geqslant 97$ & $\mathrm{~b}$ & AromaticCompounds & & \\
\hline Aldehydes & & & Benzaldehyde & $\geqslant 96$ & $\mathrm{~d}$ \\
\hline trans-2-Hexenal & $\geqslant 99$ & a & Acetophenone & $\geqslant 95$ & $\mathrm{~d}$ \\
\hline Hexanal & $\geqslant 97$ & $\mathrm{a}$ & p-Cymene & $\geqslant 97$ & $\mathrm{a}$ \\
\hline Nonanal & $\geqslant 97$ & $\mathrm{a}$ & 2-Phenylethanol & $\geqslant 97$ & d \\
\hline Furfural & $\geqslant 97$ & $\mathrm{a}$ & Cuminaldehyde & $\geqslant 97$ & $\mathrm{c}$ \\
\hline Citronellal & $\geqslant 97$ & $\mathrm{~b}$ & 1-Methylnaphthalene & $\geqslant 97$ & $\mathrm{c}$ \\
\hline (+)-Citronellal & $\geqslant 97$ & $\mathrm{~b}$ & Methylsalicylate & $\geqslant 97$ & $\mathrm{~d}$ \\
\hline Terpenes & & & 1,5-Diaminopentane & $\geqslant 99$ & e \\
\hline 2,6-dimethyl-2,4,6-octatriene & $\geqslant 99$ & $\mathrm{~b}$ & 1,4-Diaminobutane & $\geqslant 99$ & e \\
\hline$\alpha$-Terpinene & $\geqslant 97$ & c & Diethylamine & $\geqslant 97$ & $\mathrm{~d}$ \\
\hline Terpinolene & $\geqslant 97$ & $\mathrm{c}$ & Ethylenediamine & $\geqslant 97$ & $\mathrm{~d}$ \\
\hline (+)-Longifolene & $\geqslant 99$ & a & Methylamine & $\geqslant 33$ & d \\
\hline Myrcene & $\geqslant 97$ & $\mathrm{~b}$ & Ammoniasolution & $\geqslant 25$ & $\mathrm{~d}$ \\
\hline$(-)$-Limonene & $\geqslant 97$ & a & Others & & \\
\hline (-)-Camphene & $\geqslant 98$ & a & Paraffinoil & Chemicalpure & $\mathrm{f}$ \\
\hline ( \pm -Camphene & $\geqslant 98$ & $\mathrm{a}$ & & & \\
\hline
\end{tabular}

a) a, Aldrich; b, Fluka; c, Sigma; d, Beijing Medicinal and Pharmaceutical Chemistry Productions LLC; e, Tokyo Chemical Industry Co., Japan; f, TCRY. 


\subsection{Electrophysiological analysis}

Electroantennogram analysis was run using standard methods from previous experiments [13]. The antennae of 2-3 d old adult virgin moths were prepared by cutting both extremities and mounting immediately between two $\mathrm{Ag} / \mathrm{AgCl}$ electrodes filled with Kaissling saline (glucose $354 \mathrm{mmol} \mathrm{L}^{-1}$, $\mathrm{KCl} 6.4 \mathrm{mmol} \mathrm{L}^{-1}$, KH2PO4 $20 \mathrm{mmol} \mathrm{L}{ }^{-1}, \mathrm{MgCl}_{2} 12 \mathrm{mmol}$ $\mathrm{L}^{-1}, \mathrm{CaCl}_{2} 1 \mathrm{mmol} \mathrm{L}^{-1}, \mathrm{NaCl} 12 \mathrm{mmol} \mathrm{L}^{-1}$, KOH $9.6 \mathrm{mmol}$ $\mathrm{L}^{-1}, \mathrm{pH}$ 6.5). The electrode at the distal end of the antenna was connected via an interface box to a signal acquisition interface board (IDAC; Syntech, Netherlands) connected to a computer using AutoSpike software (Syntech, USA).

An air control unit (Syntech CS-05), with an outlet facing towards the antenna at a distance of $1 \mathrm{~cm}$, provided airflow at $30 \mathrm{~mL} \mathrm{~s}^{-1}$. A $10 \mu \mathrm{L}$ solution was added to a $0.5 \mathrm{~cm}$ $\times 5 \mathrm{~cm}$ piece of filter paper using a Pasteur pipette, which was fixed between air control unit and air outlet. Stimulating time was $0.1 \mathrm{~s}$ and interval time was $1 \mathrm{~min}$ to allow recovery of antennas. Chemicals were diluted using paraffin oil (concentration of $100 \mathrm{mg} \mathrm{mL}^{-1}$ ), and chemical concentrations for dose responses were: $0.1,1,10$ and $100 \mathrm{mg}$ $\mathrm{mL}^{-1}$. Doses of each stimulation were $10 \mu \mathrm{L} \times(0.1,1,10$ and $\left.100 \mathrm{mg} \mathrm{mL}^{-1}\right)=1,10,100$ and $1000 \mu \mathrm{g}$. A sample of 10 $\mu \mathrm{L}$ paraffin oil was used for the control.

Nonanal was used as the standard compound. Tests were run as the following sequence: control, nonanal, sample, control, nonanal. Each antenna was tested twice and each compound was tested with 10 different antennae per gender. To eliminate the interference of solvent and environment, electroantennogram data were adjusted using the following formula:

Electroantennogram value $=R_{\mathrm{C}}-\left(R_{\mathrm{C}-1}-R_{\mathrm{C}+1}\right) / 2$, where $R_{\mathrm{C}}$ is the raw electroantennogram value of the sample,
$R_{\mathrm{C}-1}$ is the value of the control before the sample, and $R_{\mathrm{C}+1}$ is the value of the control after the sample.

The adjusted EAG value was divided by the standard compound value to make a percentage. Mean value was tested using one-way ANOVA, and means were compared with Duncan's multiple-comparison at $P=0.05$. Each compound's mean difference of male and female moths was tested by $t$-test. All analyses were performed with the program SPSS 13.0 (SPSS Inc., Chicago, IL, USA).

\section{Results}

\subsection{Electroantennogram responses of $\boldsymbol{H}$. cunea moths to alcohols}

Electroantennogram responses of $H$. cunea moths to a spectrum of alcohols are shown in Figure 1. Female moths showed highest EAG responses to hexanol and the values were higher than controls of nonanal. There were no response differences between responses to hexanol and nonanal, but differences between hexanol and other compounds were significant $(P<0.05)$. Heptanol, cis-3-hexen-1-ol, trans2-hexen-1-ol and 2-ethyl-1-hexanol were in the second class and there was no difference among them. Bioactivities of butanol, octanol and geraniol were at a middle level and there was no difference among them. Other alcohols had low activities of less than a quarter of nonanal. Responses of female $H$. cunea moths to 1,4-pentadien-3-ol were negative $(N=30)$.

In male moths, EAG responses to hexanol were also the highest among the alcohols, but not higher than the nonanal control $(70.34 \%)$. There were significant response differences between hexanol and all other compounds, including nonanal $(P<0.05)$. Octanol was the second most active compound and showed differences with other alcohols

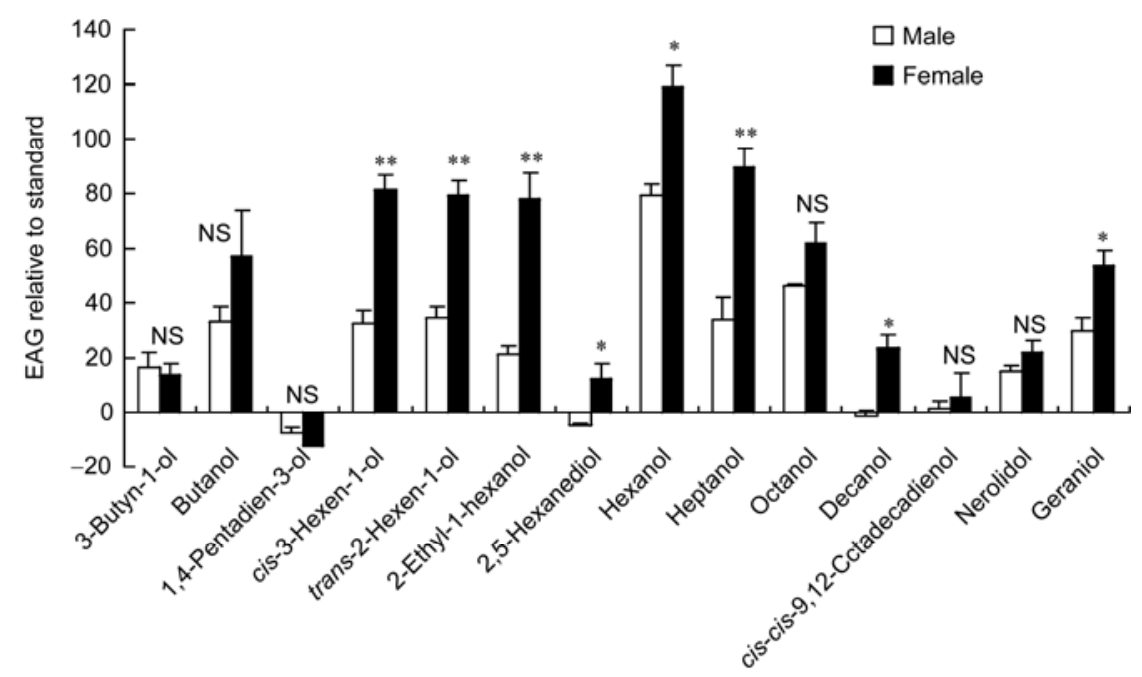

Figure 1 Mean ( \pm SE) EAG responses of female and male $H$. cunea to alcohols. NS, no significant sexual difference; $*$, significant sexual difference, $P$ $<0.05$; **, significant sexual difference, $P<0.01$, $t$-test assuming equal variances (SPSS 13.0). 
$(P<0.05)$. Other alcohols showed very low bioactivity, lower than $35 \%$ of nonanal. Responses to 1,4-pentadien-3-ol, 2,5-hexanediol and decanol were negative $(N=30)$.

\subsection{Electroantennogram responses of $\boldsymbol{H}$. cunea moths to aldehydes and acids}

As in Figure 2, female moths showed highest EAG responses to hexanal, followed by nonanal and trans-2-hexenal in decreasing order. They showed no differences among each other but there were differences between them and other aldehydes $(P<0.05)$. Furfural, citronellal and $(+)$-citronellal showed no differences among each other, but they all had a value greater than $64 \%$ of nonanal. Female moths were not sensitive to propionic acid but we cannot say categorically that females are not sensitive to all acids because we only tested a single acid in our experiment. However, our results showed that for female moths, aldehydes were more bioactive than acids.

Nonanal, hexanal and trans-2-hexenal were the three most bioactive aldehydes for male moths, and although there was no difference among them, there were significant differences between them and other aldehydes $(P<0.05)$. Furfural, citronellal and (+)-citronellal also showed no difference among each other. However, values of these three compounds were less than $45 \%$ of nonanal. Thus, male moths also showed higher activity in response to aldehydes compared with propionic acids, with the exception of furfural.

In summary, female moths showed higher activity to aldehydes than male moths. Nonanal, hexanal and trans-2hexenal were very bioactive in both genders, producing values higher than $93 \%$ of nonanal. However, propionic acids showed low activity with both genders, indicating that $H$. cunea moths were not sensitive to these acids.

\subsection{Electroantennogram responses of $\boldsymbol{H}$. cunea moths to terpenes}

As shown in Figure 3, female moths were most sensitive to 2,6-dimethyl-2,4,6-octatriene. Terpinolene was the second

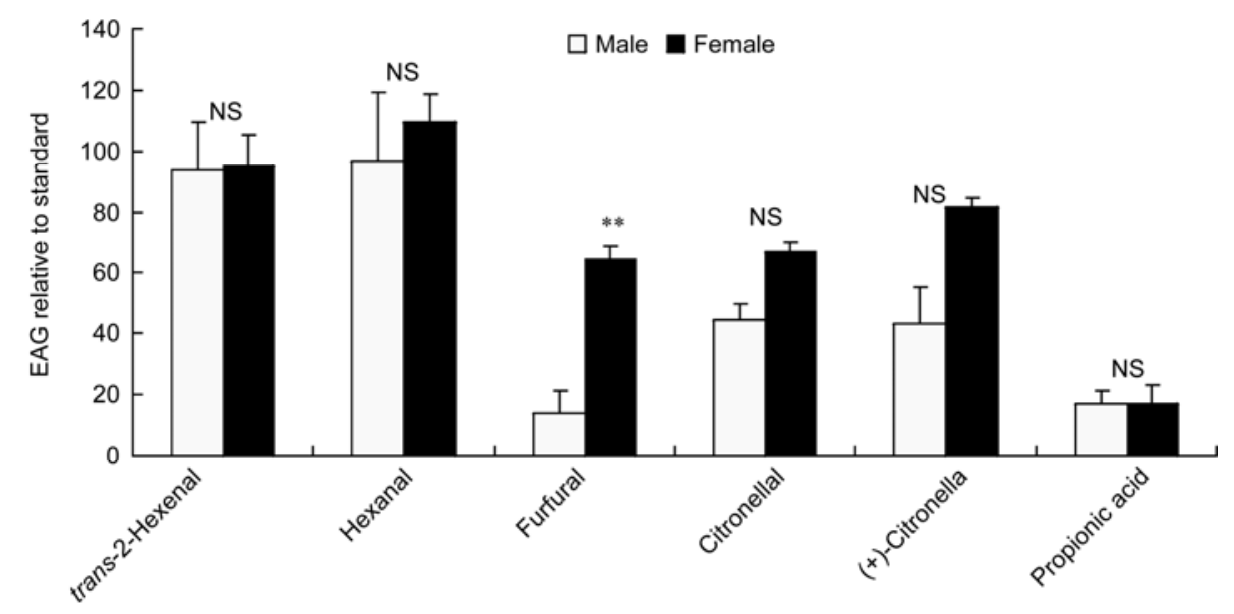

Figure 2 Mean $( \pm$ SE) EAG responses of female and male $H$. cunea to aldehydes and acids. NS, no significant sexual difference; **, significant sexual difference, $P<0.01$, $t$-test assuming equal variances (SPSS 13.0).

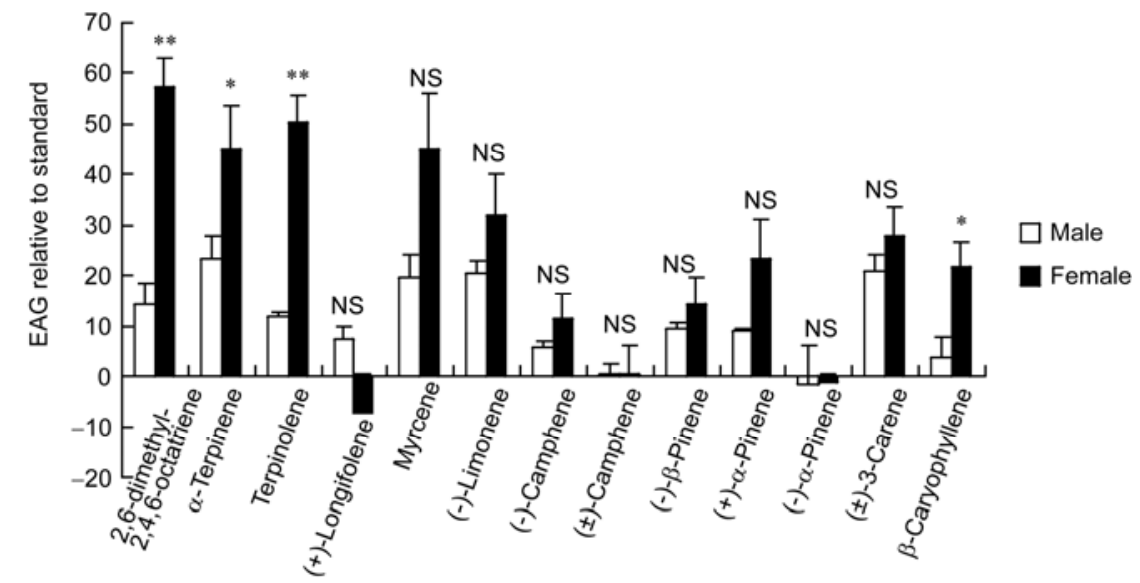

Figure 3 Mean $( \pm$ SE) EAG responses of female and male $H$. cunea to terpenes. NS, no significant sexual difference; $*$, significant sexual difference, $P<$ 0.05 ; **, significant sexual difference, $P<0.01, t$-test assuming equal variances (SPSS 13.0). 
most bioactive and there was no difference between 2,6dimethyl-2,4,6-octatriene and terpinolene. However, they were both significantly different from nonanal $(P<0.05)$. Responses to other terpenes were below $46 \%$ of nonanal, especially (+)-longifolene and (-)- $\alpha$-pinene, which both had negative values $(N=30)$.

In contrast, male moths were not very sensitive to terpenes; $\alpha$-terpinene was the most bioactive compound and it only produced an EAG response value of $23.33 \%$. Nonanal was significantly higher than all compounds $(P<0.05)$. Similarly, responses to $(-)$ - $\alpha$-pinene were also negative $(N=30)$.

Responses to 2,6-dimethyl-2,4,6-octatriene, $\alpha$-terpinene, terpinolene and $\beta$-caryophyllene were significantly different between male and female moths.

In summary, female moths were more sensitive to terpenes than were male moths. Responses to (-)- $\alpha$-pinene were negative in both genders, but $(+)-\alpha$-pinene and $(-)-\beta$ pinene were both positive. This result showed that sensitivities of $H$. cunea moths to terpenes were dependent on differing configuration of compounds.

\subsection{Electroantennogram responses of $\boldsymbol{H}$. cunea moths to esters and ketones}

As shown in Figure 4, female moths were more sensitive to esters than to ketones. Isoamyl acetate was the most bioactive and showed higher response values than nonanal, although this difference was not significant. Acetic acid cis-3hexenyl ester, ethyl acetoacetate and trans-2-hexenyl acetate were in the second class; responses of all three were higher than $51 \%$ and there was no difference among them. EAG responses to ketones were low, none being above 13\% of nonanal. Furthermore, 3-pentanone showed a negative value $(N=30)$.

Similarly, male moths also showed a higher sensitivity to esters rather than to ketones. trans-2-hexenyl acetate was the most bioactive and was different from other compounds with the exception of nonanal $(P<0.05)$. Isoamyl acetate, acetic acid cis-3-hexenyl ester and ethyl acetoacetate were in the second class with values all above $56 \%$ and no significant difference among the three compounds. Similar to female moths, responses of male moths to ketones were weak with values all below $14 \%$. Also, 3-Pentanone showed a negative value $(N=30)$.

We found that there was no significant sex difference among all compounds, except acetic acid cis-3-hexenyl ester.

In summary, $H$. cunea moths were more sensitive to esters than to ketones. Female moths were most sensitive to isoamyl acetate, but male moths were most sensitive to trans-2-hexenyl acetate. However, within each compound, there was no difference in the response between males and females.

\subsection{Electroantennogram responses of $\boldsymbol{H}$. cunea moths to aromatics}

As shown in Figure 5, in female moths, acetophenone was the most bioactive, showing EAG response values above nonanal. Though there was no difference between acetophenone and nonanal, values of acetophenone were significantly higher than all other compounds $(P<0.05)$. The next most bioactive compounds were benzaldehyde and methyl salicylate, which both had response values above $64 \%$ and no difference between each other. P-cymene was the least active, with an EAG response value lower than $17 \%$ that of nonanal.

Male moths also showed the highest sensitivity to acetophenone but only with a value of $40 \%$, which was significantly different from nonanal $(P<0.05)$ and other aromatics $(P<0.05)$. The second class contained benzaldehyde, cuminaldehyde, methyl salicylate, p-cymene and salicylic acid ethyl ester. They all showed values lower than $21 \%$ of

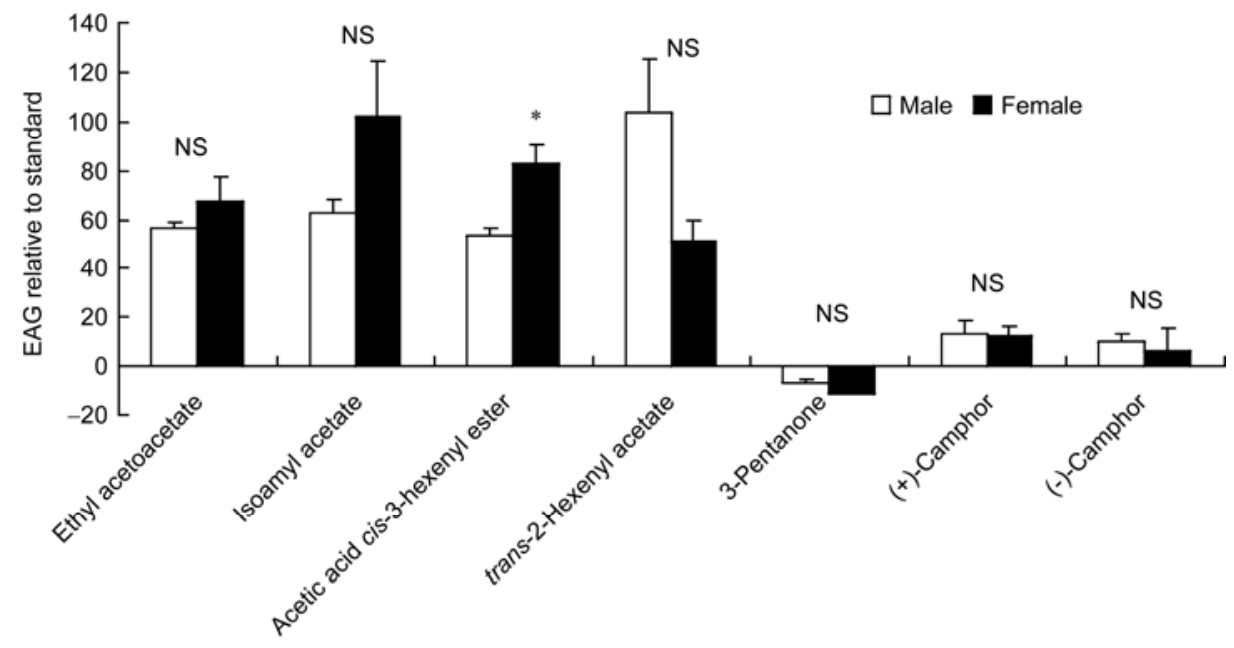

Figure 4 Mean $( \pm$ SE) EAG responses of female and male $H$. cunea to esters and ketones. NS, no significant sexual difference; *, significant sexual difference, $P<0.05$; **, significant sexual difference, $P<0.01$, $t$-test assuming equal variances (SPSS 13.0). 


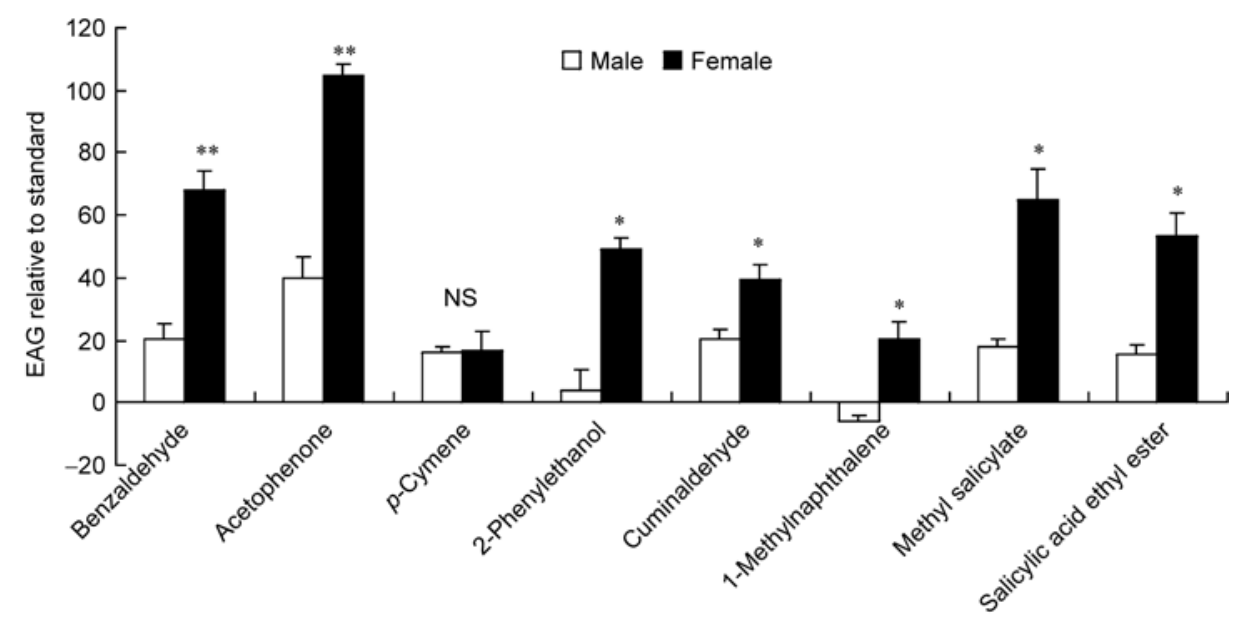

Figure 5 Mean $( \pm$ SE) EAG responses of female and male $H$. cunea to aromatics. NS, no significant sexual difference; *, significant sexual difference, $P<$ 0.05 ; **, significant sexual difference, $P<0.01, t$-test assuming equal variances (SPSS 13.0).

nonanal and no difference was found among all the five compounds. 1-Methylnaphthalene showed a negative value for male moths $(N=30)$.

With the exception of p-cymene, males and females responded significantly differently to all compounds.

In summary, female moths showed higher sensitivity to aromatics than did male moths. Acetophenone was the most bioactive for both genders. 1-Methylnaphthalene and pcymene were the least bioactive, especially 1-methylnaphthalene, which produced a negative value in male moths.

\subsection{Electroantennogram responses of $\boldsymbol{H}$. cunea moths to amines}

As shown in Figure 6, in females, 1,4-diaminobutane, 1,5diaminopentane, diethylamine and methylamine were the four most bioactive amines and were all significantly different from nonanal $(P<0.05)$ but not from each other. Re- sponses to ethylenediamine and ammonia solution were low, and values of ethylenediamine were negative for female moths $(N=30)$.

1,4-Diaminobutane showed the highest activity in males, and was significantly different from nonanal $(P<0.05)$. Male moths were not very sensitive to other amines, especially ethylenediamine, methylamine and ammonia solution, which produced negative values $(N=30)$.

With the exception of ethylenediamine and ammonia solution, there were significant sex differences in responses to amines.

In summary, all amines tested in this study showed relatively low bioactivities for both genders of moths, although female moths were more sensitive than male moths overall. Both genders of moths were most interested in 1,4-diaminobutane and had negative responses to ethylenediamine. Male moths also showed negative response values to methylamine and ammonia solution.

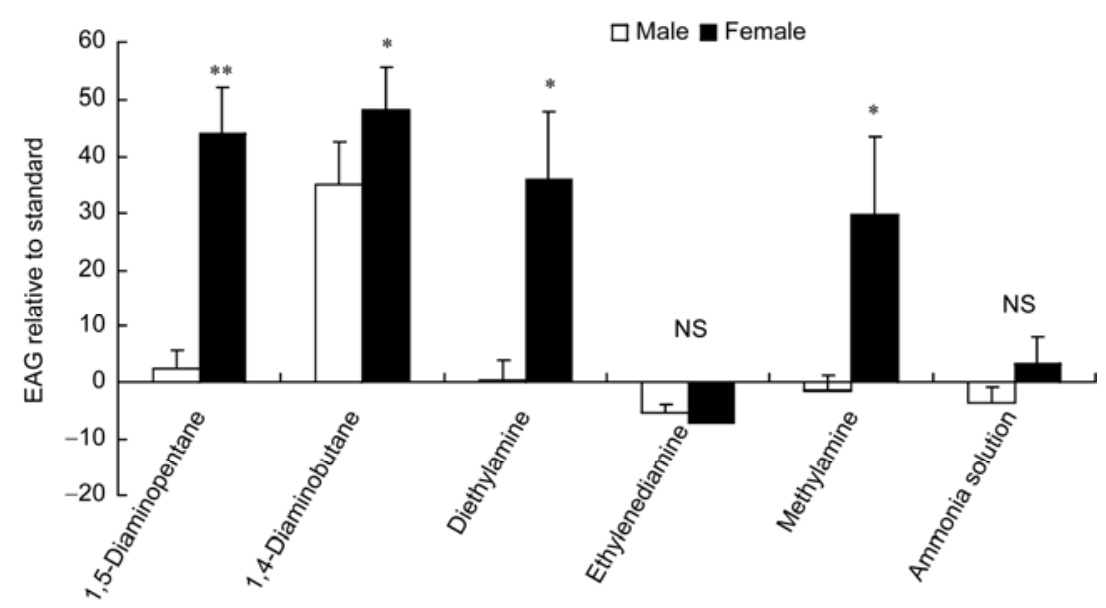

Figure 6 Mean $( \pm$ SE) EAG responses of female and male $H$. cunea to amines. NS, no significant sexual difference; *, significant sexual difference, $P<$ 0.05 ; **, significant sexual difference, $P<0.01, t$-test assuming equal variances (SPSS 13.0). 


\subsection{Dosage responses of male $\mathrm{H}$. cunea moths to selected compounds}

We selected several kinds of bioactive compounds from the experiments above, and sorted them by activity as per the sequence trans-2-hexenyl acetate $>$ nonanal $>$ hexanal $>$ trans2-hexenal $>$ hexanol $>$ isoamyl acetate>acetic acid cis-3-hexenyl ester. We conducted dosage response experiments on male moths of $H$. cunea using the 7 compounds (Table 2).

Responses of male moths to seven kinds of compounds were all positive and dose-dependent. For every compound, responses to the maximum dose of $1000 \mu \mathrm{g}$ were significantly different from those of other doses. In male moths, dose differences were not significant for $1,10,100 \mu \mathrm{g}$ doses

Table 2 EAG responses of male $H$. cunea to different doses of seven test compounds ${ }^{\text {a) }}$

\begin{tabular}{|c|c|c|}
\hline Compound & Dose $(\mu g)$ & $\begin{array}{l}\text { Relative response } \\
(\%)(\text { means } \pm \text { SE })\end{array}$ \\
\hline \multirow{4}{*}{ trans-2-Hexenyl acetate } & 1 & $4.06 \pm 1.31 \mathrm{~b}$ \\
\hline & 10 & $24.29 \pm 4.91 b$ \\
\hline & 100 & $28.82 \pm 2.98 \mathrm{~b}$ \\
\hline & 1000 & $103.84 \pm 21.64 \mathrm{a}$ \\
\hline \multirow{4}{*}{ Nonanal } & 1 & $0.16 \pm 0.38 \mathrm{~d}$ \\
\hline & 10 & $4.72 \pm 1.28 \mathrm{c}$ \\
\hline & 100 & $24.09 \pm 1.35 \mathrm{~b}$ \\
\hline & 1000 & $100 \mathrm{a}$ \\
\hline \multirow{4}{*}{ Hexanal } & 1 & $2.02 \pm 0.05 \mathrm{~b}$ \\
\hline & 10 & $10.42 \pm 0.25 b$ \\
\hline & 100 & $19.12 \pm 1.01 \mathrm{~b}$ \\
\hline & 1000 & $96.52 \pm 22.77 \mathrm{a}$ \\
\hline \multirow{4}{*}{ trans-2-Hexenal } & 1 & $7.17 \pm 2.39 b$ \\
\hline & 10 & $18.93 \pm 5.25 \mathrm{~b}$ \\
\hline & 100 & $27.00 \pm 1.78 \mathrm{~b}$ \\
\hline & 1000 & $93.62 \pm 15.89 \mathrm{a}$ \\
\hline \multirow{4}{*}{ Hexanol } & 1 & $3.95 \pm 1.20 \mathrm{c}$ \\
\hline & 10 & $9.42 \pm 0.54 \mathrm{c}$ \\
\hline & 100 & $27.88 \pm 0.46 b$ \\
\hline & 1000 & $79.34 \pm 4.37 \mathrm{a}$ \\
\hline \multirow{4}{*}{ Isoamyl acetate } & 1 & $6.53 \pm 1.64 \mathrm{c}$ \\
\hline & 10 & $9.81 \pm 2.08 \mathrm{bc}$ \\
\hline & 100 & $20.57 \pm 5.54 b$ \\
\hline & 1000 & $62.44 \pm 5.78 \mathrm{a}$ \\
\hline \multirow{4}{*}{ Acetic acid $c i s-3$-hexenyl ester } & 1 & $5.67 \pm 2.70 \mathrm{c}$ \\
\hline & 10 & $9.39 \pm 3.82 \mathrm{c}$ \\
\hline & 100 & $24.26 \pm 7.05 \mathrm{~b}$ \\
\hline & 1000 & $53.54 \pm 2.90 \mathrm{a}$ \\
\hline
\end{tabular}

a) Numbers followed by the different letters are significantly different (one-way ANOVA followed by Duncan's test at $P=0.05$ ). Standard is nonanal at dose of $1000 \mu \mathrm{g}$. of trans-2-hexenyl acetate, hexanal and trans-2-hexenal. In contrast, nonanal showed differences among all four doses. In hexanol, isoamyl acetate and acetic acid cis-3-hexenyl ester, there were no differences between 1 and $10 \mu \mathrm{g}$ doses, but these doses were significantly different from $100 \mu \mathrm{g}$ (except for isoamyl acetate, which showed no difference between 10 and $100 \mu \mathrm{g}$ ).

\section{Discussion}

We conducted electroantennogram analysis experiments using 55 kinds of compounds with male and female moths. For male moths, the most bioactive compounds were: trans2-hexenyl acetate, nonanal, hexanal, trans-2-hexenal, hexanol, isoamyl acetate, acetic acid cis-3-hexenyl ester, ethyl acetoacetate, citronellal and (+)-citronellal. For female moths, the most bioactive compounds were: hexanol, hexanal, acetophenone, isoamyl acetate, nonanal, trans-2-hexenal, acetic acid cis-3-hexenyl ester, citronellal, cis-3-hexen-1-ol, and trans-2-hexen-1-ol.

From our results, $H$. cunea moths are more likely to be interested in aliphatic derivatives, especially 6 carbon aldehydes, alcohols and esters, which are also the most common green leaf volatiles. Generally speaking, 6 carbon straight chain compounds are more bioactive than 5 carbon, 7 carbon or 8 carbon straight chain compounds [13-16]. For example, fatty alcohols can cause obvious responses in female virgin Heliothis armigera moths, and the intensity of responses are related to the length of carbon chains, which could reach a maximum value by using 1-hexanol. Burguiere et al. [17] considered that this response was not only due to the high volatility of 1-hexanol itself, but was also related to the high sensitivity of female moths to 1-hexanol. Both genders of $H$. cunea moths were more sensitive to hexanol rather than to butanol, heptanol, octanol and decanol, which proves that six carbon straight chain compounds were more bioactive than five, seven and eight carbon compounds in electroantennogram analysis experiments.

Functional groups have effects on the EAG responses of $H$. cunea moths. Male moths were more sensitive to hexanal and trans-2-hexenal rather than homologous alcohols, hexanol and trans-2-hexenol. This result proved that unsaturated compounds were more bioactive than saturated compounds [16,18-20]. Research by Burguiere et al. [17] indicated that six carbon straight chain ketones and aldehydes had lower bioactivity on female $H$. armigera moths than alcohols did. Results from the present study proved that functional groups did affect insect EAG responses, which were not positively associated with volatilities of compounds. On the other hand, female moths showed a lower sensitivity to unsaturated compounds than saturated compounds. Similar results have been reported in insect research on Lepidoptera and also Diptera [21].

Compared with aliphatic compounds, male moths were 
less sensitive to aromatics but female moths were more sensitive, especially to acetophenone, which elicited a strong response. Today, many aromatics are used as Lepidoptera attractants. For example, a mixture of benzaldehyde, phenylacetaldehyde, 2-phenylethanol and benzyl alcohol, which was released from Abelia grandiflora [22] flowers, was a strong attractant for Trichoplusia ni moths. Heath et al. [23] also reported that electrophysiological responses of Trichoplusia ni to phenylacetaldehyde were equal to responses from a mixture of benzaldehyde, benzyl acetate and phenylacetaldehyde. We suggest that acetophenone may attract female $H$. cunea moths over a long range, because it was strongly bioactive in our EAG experiments.

Terpenes were only weakly bioactive to both genders of moths. Interestingly, there was a significant difference between male and female moths to (-)- $\alpha$-pinene and ( +$)-\alpha-$ pinene, which implied that different insects may be differentially selective to configurations of chemicals. Therefore, racemic mixtures may lower the sensitivity of insects to compounds if only one kind of isomer causes an obvious response. However, most cases in nature involve mixtures of different isomers present at the same time in botanical volatiles, so there is little need to choose pure enantiomers of compounds for indoor experiments in most situations [17].

Generally, plants grown in unpleasant odors are more likely to be attacked by $H$. cunea compared with those grown in a natural environment. This phenomenon implies that unpleasant odors, such as amines, may be attractants for $H$. cunea moths. However, our EAG tests with amines indicated that none of the compounds were highly bioactive, and it is possible that bioactive compounds of $\mathrm{H}$. cunea are mixtures of several compounds instead of a single component, as is the case for Phthorimaea operculella moths [24] locating hosts by a blending of different signal chemicals. Many herbivorous insects locate hosts by the mixture of different chemicals released by the plants [19], such as Rhagoletis pomonella being attracted by the five component mixture of butyricacidbutylester, propyl caproate, butyl caproate, butyric acid hexylester and amyl caproate [25]. The green leaf volatile mixture of trans-2-hexenal, cis-3-hexe1-ol, trans-2-hexe-1-ol and acetic acid cis-3-hexenyl ester is an odor signal for Leptinotarsa decemlineata to locate hosts $[13,20,26]$, so it is likely that $H$. cunea moths also use a mixture of chemicals for host location.

Our results indicate that female moths were more sensitive than male moths to a spectrum of plant chemical compounds, which accords with results from past studies [4,25,27-30]. Because of oviposition, female moths may be more sensitive to botanical odors to find better places for breeding. Furthermore, herbivore induced volatiles can also have effects on female oviposition behaviors [31]. This phenomenon may be caused by differential quantities of olfactory sensation receptors in male and female of insects [29].

Green leaf volatiles are considered to be varieties of kairomones [32]. Many studies have indicated that olfactory reactions of male insects can be heavily affected by leaf volatiles $[5,6,15,33]$. When combined, botanical secondary substances and sex pheromones could attract Helicoverpa armigera moths over large distances [34]. Male moths of $H$. cunea had strong responses to chemicals of trans-2-hexenyl acetate, nonanal, hexanal, trans-2-hexenal, hexanol, isoamyl acetate and acetic acid cis-3-hexenyl ester, which implies that these compounds may stimulate sex pheromone receptors and enhance the efficiency of sex pheromones. Botanical chemicals comprised a large part of the compounds found to be bioactive for $H$. cunea moths, and most of them were considered herbivore or oviposition induced secondary substances $[35,36]$. Functions of induced botanical secondary substances are complicated and are usually considered to be related to plant defense reactions $[35,36]$. However, volatiles can also attract certain species of insects, including parasitic wasps [37]. Invasion and mating of $H$. cunea in China may be related to the combined effects of sex pheromones and plant volatiles. Moreover, sensitivities of moths to herbivore induced odors indicates $H$. cunea can potentially have a wider range of hosts and a better adaptability than local insects, which will further increase the invasive ability of this pest. We suggest that botanical volatiles with bioactive properties could be used as synergists to increase the efficiency of $H$. cunea sex pheromones, and consequently reduce costs of monitoring of $H$. cunea moths.

This work was supported by National Basic Research Program of China (2009CB119204).

1 Kesselmeier J, Staudt M. Biogenic volatile organic compounds (VOC): An overview on emission, physiology and ecology. J Atmos Chem, 1999, 33: 23-88

2 Howard R W, Blomquist G J. Ecological, behavioral, and biochemical aspects of insect hydrocarbons. Annu Rev Entomol, 2005, 50: 371-393

3 Landolt P J. Host plant influences on sex pheromone behavior of phytophagous insects. Annu Rev Entomol, 1997, 42: 371-391

4 Light D M, Flath R A, Buttery R G, et al. Host-plant green-leaf volatiles synergize the synthetic sex pheromones of the corn earworm and codling moth (Lepidoptera). Chemoecology, 1993, 4: 145-152

5 Dickens J C, Visser J H, Van-Der-Pers J C N. Detection and deactivation of pheromone and plant odor components by the beet armyworm, Spodoptera exigua (Hübner) (Lepidoptera: Noctuidae). J Insect Physiol, 1993, 39: 503-516

6 Dickens J C, Smith J W, Light D M. Green leaf volatiles enhance sex attractant pheromone of the tobacco budworm, Heliothis virescens (Lep. Noctuidae). Chemoecology, 1993, 4: 175-177

7 Su M W, Fang Y L, Tao W Q, et al. Identification and field evaluation of the sex pheromone of an invasive pest, the fall webworm $\mathrm{Hy}$ phantria cunea in China. Chin Sci Bull, 2008, 53: 555-560

8 Hill A S, Kovalev B G, Nikolaeva L N, et al. Sex-pheromone of the fall webworm moth, Hyphantria cunea. J Chem Ecol, 1982, 8: 383-396

9 Reddy G V, Guerrero A. Interactions of insect pheromones and plant semiochemicals. Trends Plant Sci, 2004, 9: 253-261

10 Travis H J. The effect of eastern tent caterpillar (Malacasoma americanum) infestation on fall webworm (Hyphantria cunea) selection of black cherry (Prunus serotina) as a Host Tree. Am Midl Nat, 2005, 153: $270-275$ 
11 D'alessandro M, Turlings T C J. Advances and challenges in the identification of volatiles that mediate interactions among plants and arthropods. Analyst, 2006, 131: 24-32

12 Li W G, Zhang L W, Wang C, et al. Analysis of mulberry leaf volatile components by static headspace-gas chromatography-mass spectrometry (in Chinese). Canye Kexue, 2009, 35: 355-361

13 Visser J H. Electroantennogram responses of the Colorado beetle, Leptinotarsa decemlineata, to plant volatiles. Entomol Exp Appl, 1979, 25: 86-97

14 Kozlowski M W, Visser J H. Host plant related properties of the antennal olfactory system in the oak flea weevil, Rhynchaenus quercus. electroantennogram study. Entomol Exp Appl, 1981, 30: 169-175

15 Dickens J C. Olfaction in the boll weevil Anthonomus grandis Boh. (Coleoptera Curculionidae) electroantennogram studies. J Chem Ecol, 1984, 10: 1759-1786

16 Light D M, Jang E B, Dickens J C. Electroantennogram responses of the mediterranean fruit fly, Ceratitis capitata, to a spectrum of plant volatiles. J Chem Ecol, 1988, 14: 159-180

17 Burguiere L, Marion-Poll F, Cork A. Electrophysiological responses of female Helicoverpa armigera (Hübner) (Lepidoptera; Noctuidae) to synthetic host odours. J Insect Physiol, 2001, 47: 509-514

18 Visser J H. Differential sensory perception of plant compounds by insects. Am Chem Soc Symp Ser, 1982, 208: 215-230

19 Visser J H. Host odour perception in phytophagous insects. Ann Rev Entomol, 1986, 31: 121-144

20 Thiery D, Visser J H. Masking of host plant odour in the olfactory orientation of the Colorado potato beetle. Entomol Exp Appl, 1986, 41: $165-172$

21 Guerin P M, Stadler E. Host odour perception in three phytophagous Diptera. A comparative study. In: Visser J H, Minks A K, eds. Proceedings of the Fifth International Symposium on Insect-Plant Relationship, Purdoc, Wageningen, 1982. 95-105

22 Haynes K F, Zhao J Z, Latif A. Identification of floral compounds from Abelia grandiflora that stimulate upwind flight in cabbage looper moths. J Chem Ecol, 1991, 17: 637-647

23 Heath R R, Landolt P J, Dueben B, et al. Identification of floral compounds of night-blooming jessamine attractive to cabbage looper moths. Environ Entomol, 1992, 21: 854-859

24 Das P D, Raina R, Prasad A R, et al. Electroantennogram responses of the potato tuber moth, Phthorimaea operculella (Lepidoptera Gel- ichiidae) to plant volatiles. J Biosci, 2007, 32: 339-349

25 Zhang A J, Linnjr C, Wright S, et al. Identification of a new blend of apple volatiles attractive to the apple maggot, Rhagoletis pomonella. J Chem Ecol, 1999, 25: 1221-1233

26 Visser J H, Ave D A. General green leaf volatiles in the olfactory orientation of the Colorado potato beetle, Leptinotarsa decemlineata. Entomol Exp Appl, 1978, 24: 538-549

27 Van-Der-Pers J N C. Comparison of electroantennogram response spectra to plant volatiles in seven species of Yponomeuta and in the tortricid Adoxophyes orana. Entomol Exp Appl, 1981, 30: 181-192

28 Ramachandran R, Khan Z R, Caballero P, et al. Olfactory sensitivity of two sympatric species of rice leaf folders (Lepidoptera Pyralidae) to plant volatiles. J Chem Ecol, 1990, 16: 2647-2667

29 Raguso R A, Light D M, Pickersky E. Electroantennogram responses of Hyles lineata (Sphingidae Lepidoptera) to volatile compounds from Clarkia breweri (Onagraceae) and other moth-pollinated flowers. J Chem Ecol, 1996, 22: 1735-1767

30 Malo E A, Cruz-López L, Toledo J, et al. Behavioral and electrophysiological responses of the Mexican fruit fly (Diptera: Terphritidae) to grava volatiles. Fla Entomol, 2005, 88: 364-371

31 Hare J D. Ecological role of volatiles produced by plants in response to damage by herbivorous insects. Annu Rev Entomol, 2011, 56: 161-180

32 Ruther J, Reinecke A, Hilker M. Plant volatiles in the sexual communication of Melolontha hippocastani response towards time-dependent bouquets and novel function of ( $Z$ )-3-hexen-1-ol as a sexual kairomone. Ecol Entomol, 2002, 27: 76-83

33 Dickens J C. Green leaf volatiles enhance aggregation pheromone of the boll weevil Anthonomus grandis. Entomol Exp Appl, 1989, 52: 191-203

34 Lu Y J, Zhang X X. Effects of interaction of plant volatiles and sex pheromone on EAG response of Helicoverpa armigera (Huebner) (in Chinese). Acta Ecol Sin, 2003, 23: 308-313

35 Kessler A, Baldwin I T. Defensive function of herbivore-induced plant volatile emissions in nature. Science, 2001, 291: 2141-2144

36 Kessler A, Baldwin I T. Plant responses to insect herbivory: The emerging molecular analysis. Annu Rev Plant Biol, 2002, 53: 299328

37 Dicke M, Hilker M. Induced plant defences: From molecular biology to evolutionary ecology. Basic Appl Ecol, 2003, 4: 3-14

Open Access This article is distributed under the terms of the Creative Commons Attribution License which permits any use, distribution, and reproduction in any medium, provided the original author(s) and source are credited. 